\title{
Lightweight and structure optimization of wheel body of the large hoisting sheave
}

\author{
HuiLiang Li YuCui Jiao \\ Zhengzhou University of Industrial Technology,Zhengzhou 451100, China
}

\begin{abstract}
Keywords: Wheel body of the hoisting sheave; statics analysis; deformation
Abstract:To ensure wheel body of the hoisting sheave strength and stability condition. For the purpose of wheel body lightweighting. There are two schemes to reduce body weight.Reduce the spokes at the same time increase the ring stiffened plate, and reduce the spokes at the same time change the spokes width and thickness. The wheel body was established based on Pro/E 3D geometric model. Import the mesh in the Workbench of ANSYS software for finite element model. Statics analysis to select the optimized scheme. Establish a hoisting sheave wheel body under the actual working condition of widening the width - deformation - wheel weight relational table. Analysis to lightweight at the same time ensure that stiffness of wheel,then it can obtaine the optimal result.
\end{abstract}

\section{Introduction}

In recent decades, many scholars of the day wheel system attaches great importance to carry out a number of related theoretical studies.But mostly theoretical formula established on the basis of a large number of simplified formula tedious and error is relatively large, can not properly guide the design and development work sheave system.By the sheave system finite element analysis to identify sheave body deformation in the actual working conditions, it can provide important theoretical basis for weight reduction and structural optimization sheave wheel body.

Sheave is an important facility in the hoisting system, because it is located high above the well tower, inspection, maintenance, replacement extremely difficult and inconvenient. Currently sheave generally useful life of more than 20 years, the strength and stability have some protection, but its weight far exceeds the weight of overseas sheave system, apparently in ensuring the strength and stability of the sheave premise, this Research is an important thing for the weight and waste materials to reduce the sheave system[1].

\section{Sheave wheel stress analysis}

In this paper, JKMD-5.7 $\times 4$ (IV) type multi-rope friction hoist sheave system for the study, which has two sets of sheave system, their working conditions and force are identical, and therefore only one group for analysis can, according to the number of hoist rope and pitches, each corresponding to the shaft equipped with four sheaves, one of which is fixed sheave, connected by a bond with the day fixed axle, the other three for the swimming sheave, swimming sheave relative rotation of the shaft, thereby eliminating the pieces of rope lifting speed difference of sheave rope grooves of sliding wear. Each sheave force identical, where the force of a wheel diagram shown in Figure 1. Each wheel spokes day at work to withstand the repeated alternating load in the wrap-around angle range under pressure around the outside of the package by pulling most of the angular range. In addition, the spokes in the rope grooves produce bending plane of symmetry[2].

\section{Create a finite element model of the sheave}

Sheave system is a large assembly, it is not considered contacting relationship between the sheave during analysis, Fixed sheave and swimming sheave subject to the same stress situation, so there is only one sheave to simulate round, This result does not affect the analysis of the effectiveness and accuracy. The establishment of a sheave model uses Pro / E three-dimensional modeling software, shown in Figure 2. 


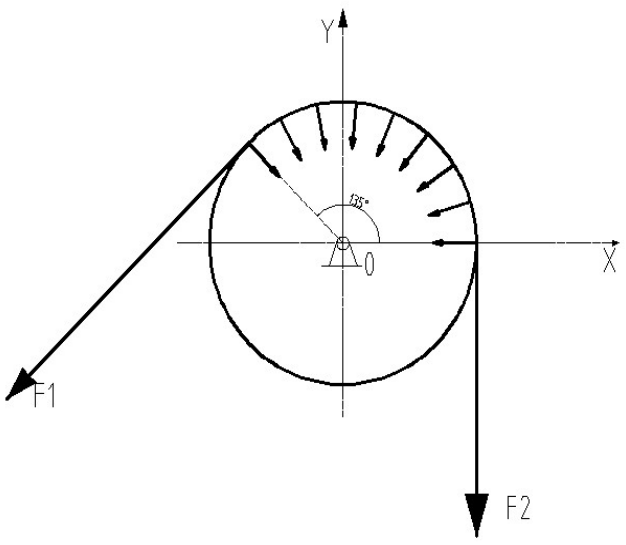

Fig.1 Force of the hoisting sheave

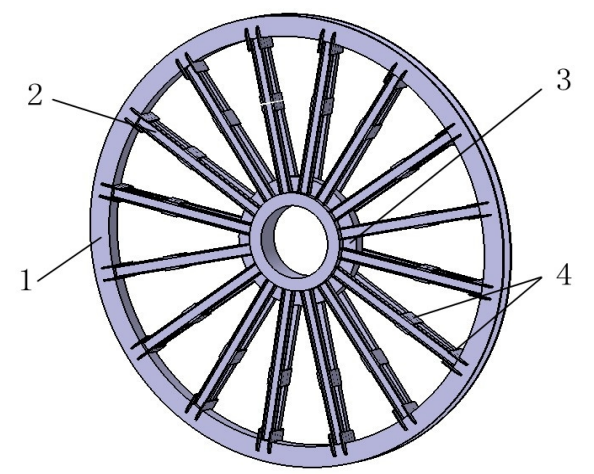

1- rim 2 - spokes 3- wheels 4- steel Fig. 2 The 3D model of wheel body

\section{Sheave model static analysis}

Statics of sheave analyzed using Workbench, Sheave deformed mainly in two parts: 1. undertake the wheel load of presents concave surface of the wire rope deformation, maximum deformation at the edges of the rope wrap angle. 2. Because under pressure radial spokes and tangential tension and showing distortion, deformation occurs mainly on the face perpendicular to the central axis. For more than two variants of the site, two programs were designed to optimize the structure of the day wheel, strengthening more than two stiffness, reducing the two-day round spokes in ensuring the rigidity of the premise in order to achieve the purpose of weight reduction[3].

Optimization Option 1: Reduce two spokes, while increasing the annular rib plate.

Optimization Option 2: Reduce two spokes, spoke while changing the thickness and width.

\section{Programme Selection}

Using two optimization statics analysis of normal conditions in the wheel sheave system,Deformation in Figures 3 and 4.

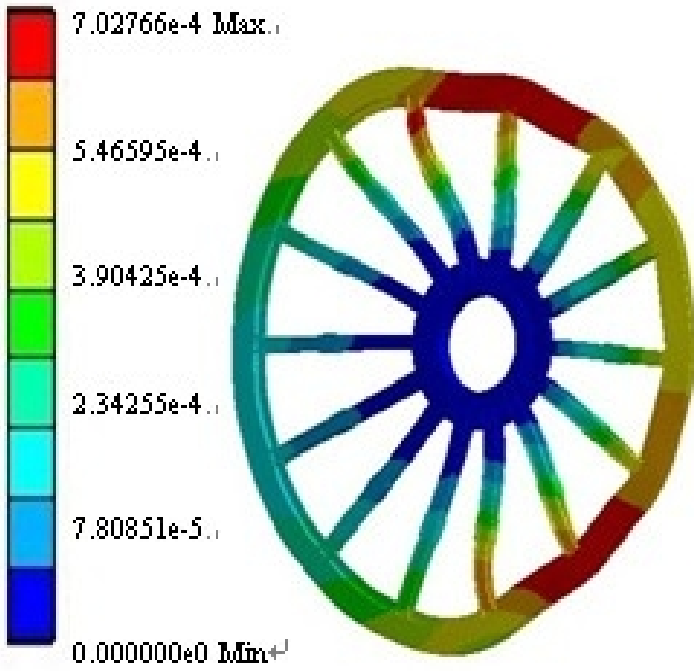

Fig.3 The static analysis of deformation of optimization 1 model

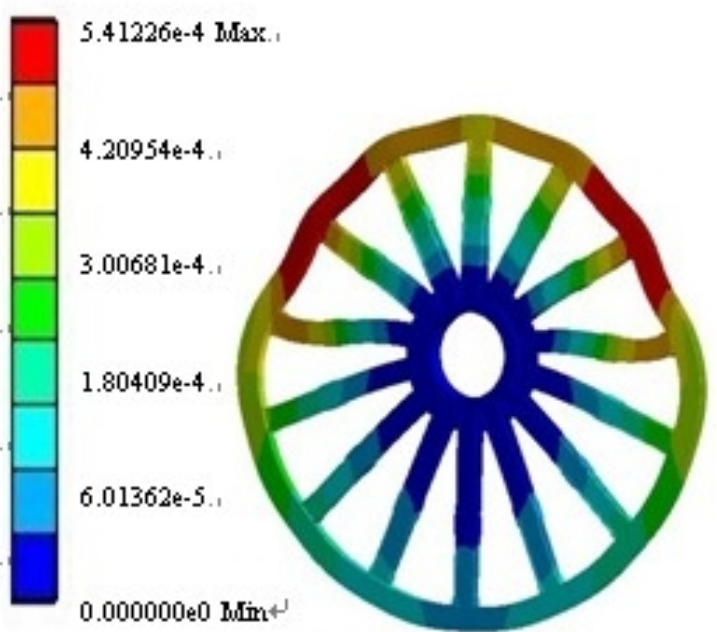

Fig.4 The static analysis of deformation of optimization 2 model 
By static simulation, the original sheave wheel, optimization scheme 1 and 2 of each model - the amount of deformation - the relationship between weight change results sheave wheel as shown in table 1 .

Tab1 The models-deformation - head sheave wheel body weight table

\begin{tabular}{|c|c|c|}
\hline Model & $\begin{array}{c}\text { Amount of } \\
\text { deformation }(\mathrm{mm})\end{array}$ & Round body weight $(\mathrm{kg})$ \\
\hline $\begin{array}{c}\text { Original sheave } \\
\text { wheel }\end{array}$ & 0.5176 & 7462 \\
\hline $\begin{array}{c}\text { Optimization } \\
\text { Scheme 1 }\end{array}$ & 0.7028 & 7276 \\
\hline $\begin{array}{c}\text { Optimization } \\
\text { Scheme 2 }\end{array}$ & 0.5412 & 7221 \\
\hline
\end{tabular}

From Table 1, in Scheme 1, although reducing the weight sheave, but the deformation significantly increased, indicating that the rim inner rib by increasing the overall stiffness enhancing effect is not obvious, Option 2 to reduce the weight sheave under the premise, less the amount of deformation increases, indicating that by changing the parameters of the spokes, the structure may be more significant to achieve light weight and stiffness optimization. In summary, the parameters can be the result of the spokes, the structure was further optimized in Scheme 2.

Deformation in the spoke direction with reference to Figure 3, the parameters need to be optimized is a plane perpendicular to the axial stiffness, increase the width of the spokes can significantly enhance the stiffness in this direction[4]. Taking into account the small axial deformation of the spokes, the spokes will reduce $5 \mathrm{~mm}$ thickness to reduce weight, and therefore the width of the spokes and static variables to optimize simulation analysis, specific widened width - the amount of deformation - sheave wheel weight changes in the relationship between the results shown in table 2.

Tab2 Widening the width - Deformation - Sheave wheel body weight able

\begin{tabular}{|c|c|c|}
\hline WideningWidth (mm) & $\begin{array}{c}\text { Amount of } \\
\text { deformation }(\mathrm{mm})\end{array}$ & Round body weight (kg) \\
\hline 90 & 0.5541 & 7170 \\
\hline 100 & 0.5412 & 7221 \\
\hline 110 & 0.5279 & 7272 \\
\hline 120 & 0.5157 & 7322 \\
\hline 130 & 0.5035 & 7373 \\
\hline
\end{tabular}

Table 2 shows, with the increase of the width of the spokes, sheave weight linearly increased while the amount of deformation sheave decreases linearly. Safety factor because the original program design selected large, so the amount of deformation sheave requirements may be appropriate to allow a small amount of the increase, taking into account the purpose of lightweight, you can select the day to increase the width of the wheel spokes $110 \mathrm{~mm}$ solution for the optimal solution. This scheme makes sheave wheel body weight $190 \mathrm{Kg}$, the amount of deformation increases only $0.01 \mathrm{~mm}$, the results achieved weight reduction requirements while ensuring the rigidity sheave wheel body. 


\section{summary}

This paper established a multi - rope friction hoist sheave geometry body and analyze its statics, three models come deformation, and its optimization analysis. Sheave body should be a reasonable addition to the rope groove, the most important parameter is the number of spokes. Sheave body ideal structure type, there is bound to be the best number of spokes and spoke structure, only through continuous optimization analysis to find the best combination of fashion.

(1) The finite element method can get more accurate and intuitive deformation distribution of the wheel, the traditional method can only be made with plenty of rough calculation in a simplified manner.

(2) This is a reasonable result using the finite element model of static analysis. Can improve efficiency of the analysis to give each program model - the amount of deformation - sheave wheel body weight relationship, it can enhance the purpose of the analysis and optimization.

(3) The spokes stiffness is one of the main factors affecting the overall stiffness of the sheave, its axial rigidity is enhanced significantly reduce the amount of deformation day round.

\section{References}

[1] Fan. Cheng-you. Coal mine during multi rope friction hoisting equipment type selection design[J]. Coal Technology, 2013 (07):31-32.

[2] HAN D T, GE S R. Frictional heat analysis of mine hoist and Numerical simulation on temperature field of gasket[J]. Mining Science and Technology. 2009, (19): 0040-0044.

[3] MA J Q, LI G J. Reliability analysis of slope with Latin hypercube sampling and K-S test[J]. Academia Sinica, 2011, 32(7): 2153-2156.

[4] Kim H k, Kim C, Bae W B. Development ofoptimization technique ofwarm shrink fitting process for automotive transmission parts(3D FE analysis)[J]. Journal of Materials Processing Technology, 2007, 187: 458-462. 